\title{
Effects of three methods of breast milk expression on breastfeeding initiation, breast milk extraction and proportion of exclusive breastfeeding-an open label, randomized controlled trial
}

\section{Yun Zhou}

Shanghai Jiaotong University School of Medicine Xinhua Hospital

Jiaying Ni

Shanghai Jiaotong University School of Medicine Xinhua Hospital

Chenchen Zhou

Shanghai First Maternity and Infant Hospital

\section{Ying Chen}

Shanghai General Hospital Jiading Branch

Julian Little

University of Ottawa Faculty of Medicine

Lin Zhang ( $\sim$ lily_yulin2003@msn.com)

International peace maternity and child health hospital https://orcid.org/0000-0002-2728-4715

\section{Research}

Keywords: Breast milk expression, Breastfeeding initiation, Maternal-infant separation, Exclusive breastfeeding, Randomized controlled trial

Posted Date: June 16th, 2020

DOI: https://doi.org/10.21203/rs.3.rs-35741/v1

License: (c) (i) This work is licensed under a Creative Commons Attribution 4.0 International License. Read Full License 


\section{Abstract}

Background Direct breastfeeding hasmultiple health benefits. Some mothers need to express their breasts when directly breast feeding is unavailable after delivery . However, there is insufficient evidence regarding an effective method of breast milk expression in the absence of an infant's suction. We aimed to investigate the effects of different methods of breast milk expression on breastfeeding initiation, efficacy of breast milk extraction, and on the rate of exclusive breastfeeding at 6 months.

Methods In this open-label, randomized controlled trial, the participants of mother-infant dyads separated immediately after birth for at least 6 hrs were recruited and randomly assigned (1:1:1) into three groups: (1) hand expression of breast milk; (2) sequential electrical breast pumping: and (3) simultaneous electrical breast pumping. The two primary outcome measures were the time required for initiation of breastfeeding and the milk volume expressed in a 10-minute session (direct breastfeeding in hand expression group) at 3 days and at 6 weeks; a secondary outcome was the proportion of exclusive breastfeeding at 4 and 6 months. Analysis was according to intention-to-treat.

Results Between 2018 and 2019, we recruited 226 participants, of whom 154 were randomized. The time to breastfeeding initiation showed no difference between the three groups. At 3 days and at 6 weeks, the volume of breast milk produced by 10 minutes of sequential pumping or simultaneous pumping was less than that of hand pumping before direct breastfeeding, while the volume did not differ significantly between the two pumping groups. However, simultaneous breast pumping was more effective than sequential pumping both in volume and saving time in women who had established breastfeeding. The rate of exclusive breastfeeding at 4 months was $21.0 \%$, and at 6 months $10.6 \%$, and did not differ between the three methods of breast milk expression.

Conclusions Hand expression, sequential breast pumping, or simultaneous breast pumping in absence of immediate infant suction after birth did not show differences on the time required to initiate breastfeeding, or on the rate of exclusive breastfeeding at 4 and 6 months.

Trial registration This trial was registered with www.chictr.org.cn (ChiCTR1800018193) on 4 September 2018.

\section{Background}

Breastfeeding provides natural, and is considered unequivocally to be the optimal nutrition for infants. ${ }^{1,2}$ Indeed, the World Health Organization (WHO) recommends exclusive breastfeeding for at least the first six months of life and continued with appropriate introduced supplement food for up to two years and beyond. ${ }^{3}$ The health benefits of breastfeeding include prevention of infant death, ${ }^{4-6}$ of diseases such as otitis media, atopic eczema, and possibly allergic rhinitis in early life, ${ }^{1,7}$ and of non-communicable diseases such as obesity, diabetes and heart diseases in later life ${ }^{8-10}$ enhancement of early childhood development, especially neurodevelopment, as reflected by increased IQ scores and better school 
performance; ${ }^{11}$ and reduction of maternal risks of postpartum hemorrhage, breast and ovarian cancer, and some cardiovascular diseases. ${ }^{1,12-16}$ In addition, breastfeeding is considered to be a socioeconomic lever to mitigate inequities in provision of health service resources. ${ }^{17}$

There are a number of circumstances in which initiating breastfeeding soon after birth ( $<1 \mathrm{hr})$ and sustaining exclusive breastfeeding are not possible. First, premature infants have difficulties in latchingon, sucking and digestion, ${ }^{18,19}$ yet provision to these infants with their own mothers' milk is important ${ }^{2}$. Compared with full-term mothers, mothers of preterm infants find it more difficult to establish and sustain lactation, ${ }^{20}$ it has been reported that only about $50 \%$ of them are able to express sufficient milk for their infants. ${ }^{21}$ Unless within $1 \mathrm{hr}$ of delivery, breast expressing with pump did not promote lactogenesis stage II among mothers with premature infants. ${ }^{22-24}$ Second, mothers who deliver by cesarean section are usually prevented from putting their infants to breast $<1 \mathrm{hr}$ after birth, and hence have a delay in lactation initiation and reduced lactation duration. ${ }^{25-33}$ In view of the high annual Cesarean delivery rates reported in mainland of China, raised from $28.8 \%$ in 2008 to $34.9 \%$ in $2014,{ }^{34}$ together with the short period (14 weeks) of paid maternal leave, ${ }^{35}$ the prevalence of breast pumping expression would be expected to be high in China. For mothers of established breastfeeding it was believed that the excessive breast milk after direct breastfeeding should be removed and the breast milk should be expressed regularly in absence of direct breastfeeding in order to stimulate the breast milk production. ${ }^{19,27,36-38}$ As a result, the breast expressing was recommended to nursing mothers in absence of infant suction after birth or after established lactation in context-specific manner.

Survey data indicated that the rate of breast milk expressing could reach $85 \%$ before 4 months in Australia women ${ }^{39}$. Apart from scenarios that necessitate expression of breast milk, there is evidence that many women with full-term, healthy infants also express breast milk regularly. Among Australian women with healthy term infants $60 \%$ had a breast pump within two days postpartum and $40 \%$ of them expressed several times a day in the following two weeks. ${ }^{40}$ Among Chinese women with healthy term infants $64 \%$ used a breast pump and $22 \%$ expressed exclusively at 6 weeks postpartum ${ }^{41}$. The undeserved prominence of breast milk expressing prevalence even drove the evolution of the definition of exclusive breastfeeding by including both at the breast and with exclusively pumped milk, though its potential consequence is unknown. ${ }^{42,43}$ On the other hand, bottle-feeding the full-term, healthy infants with expressed milk (exclusively pumped in this case) did not contribute to the rate and duration of exclusive breastfeeding. ${ }^{23,41,44,45}$ Breast pumping should not be used in full-term, healthy birth as stated in the fourth step of the revised "Ten steps to successful breastfeeding" guidance that "Facilitate immediate and uninterrupted skin-to-skin contact and support mothers to initiate breastfeeding as soon as possible after birth" [https://www.who.int/nutrition/bfhi/ten-steps/en/]. With regard to methods of breast expression, a systematic review concluded that, depending on the purpose of milk expression, hand expressing may be as effective as electric pumping but there was considerable heterogeneity between studies and potential bias arising from commercial involvement in about half of the included studies. ${ }^{46}$ Thus, the effectiveness of different methods of breast pumping remains unclear. 
We therefore undertook a randomized controlled trial of the effect of alternative methods of breast pumping on time required to initiate lactation, volume of milk production and rate of direct breastfeeding in otherwise healthy mother-infant pairs in which the neonates had been separated from their mothers immediately after birth for at least 6 hrs to undergo medical observation or examination. Three typical methods in breast milk expressing were included in this trial, namely hand expressing (different from vacuum sucking, usually positive pressure without any extra gadget; in addition, breast massage is common in women before or after colostrum appearance), sequential suction (hand pressing or electronic pumping), simultaneous suction (which mimics the infant's suction behavior by incorporating an extra stimulating module before sucking and using the rhythm of vacuum suction close to the frequency of infant's sucking).

\section{Methods}

\section{The aim}

To investigate the effects of different methods of breast milk expression on breastfeeding initiation, efficacy of breast milk extraction, and as a consequence, on the rate of exclusive breastfeeding at 6 months.

\section{Design and setting of the study}

In this open-label, randomized controlled trial, the participants were recruited at Xinhua Hospital affiliated to Shanghai Jiaotong University, a hospital certified as a Baby Friendly Hospital. This is a tertiary general hospital with a 75-bed obstetrics unit and approximately 3300-3500 deliveries annually. Being the Shanghai Critical Maternal Transfer Center, Shanghai Prenatal Diagnosis Center, and Shanghai Premature Birth Center, it is also a referral centre for high-risk pregnancies from other hospitals in Shanghai City and surrounding provinces.

\section{Participants}

The participants were recruited from Sept. 2018 to Sept. 2019 through posters at an Obstetrics unit and in a delivery room of the hospital. Mother-neonate dyads were potentially eligible if the mother was aged 2040 years, had attended antenatal education course in the hospital, committed to direct breastfeeding for at least 4 months in her maternity leave and were willing to express breast milk if required. The neonates had to have been delivered at gestational age $\geq 34$ weeks and body weight $\geq 2000$ gram with 1-minute Apgar score $\geq 7$. Dyads in which the woman had a history of smoking or regular alcohol drinking, or contraindications (such as human immunodeficiency virus infectious diseases) to direct breastfeeding, or the infant had congenital anomalies or was unsuitable for direct breastfeeding, were excluded. Ethical approval of this trial was obtained from the Institutional Review Board of Shanghai Jiaotong University School of Medicine. All participants provided written informed consent before any trial-related procedures, first during antenatal preparation classes in the $30^{\text {th }}-37^{\text {th }}$ weeks of gestation, and then if the motherinfant dyads matched the eligibility criteria, just before delivery or within 2 hours after delivery, depending 
on the clinical situation of the baby. Since participants recruitment the principles of quality control were followed. This trial is registered with Chinese Clinical Trial Registry (http://www.chictr.org.cn), code ChiCTR1800018193. The reporting of the trial is guided by the extension to the CONSORT statement for multi-arm parallel-group randomized trials. ${ }^{47}$

\section{Randomization and masking}

Participants were randomly assigned into three groups (1:1:1) through a simple "lottery drawing" process without replacement. The lottery of grouping information, pre-sealed in 155 opaque envelops, was opened in the face of two investigators. The three groups were defined according to the methods of breast milk expression in the absence of infant sucking, namely hand expression, sequential pumping (Avent SCF902/11 single electric breast pump, Philips Invest, China) and simultaneous pumping (Avent SCF303/01 bilateral electric breast pumps, Philips Invest, China) in the first 6 months after birth.

This is an open-label trial. The diary check, coding, questionnaire information collection will be conducted by two nurses who did not know the allocation. The groups are coded that will not suggest any clue for electric breast pump usage and the statistician will not know the information of groups in prior.

\section{Procedures}

Participants were interviewed face-to-face during antenatal preparation classes before recruitment. Actual recruitment and simple randomization occurred immediately (<30 min) after birth at bedside of delivery/recovery room that the decision was made based on whether the neonates should be separated from their mothers for $\geq 6$ hours. The common causes of mother and infant separation include preterm birth, neonatal asphyxia, maternal or neonatal suspicious infections (such as maternal fever before delivery, meconium-stained amniotic fluid) and complications of delivery. Physical examination and laboratory tests in pregnancy were carried out and demographic data were obtained.

All participants were trained in three manual expression of breast milk during mother-baby separation, according to guidelines for initiating milk supply in the absence of infant suction. ${ }^{48}$ Women were asked to begin breast milk expression at 2 hours after birth and continue with an interval of 2-3 hours between subsequent sessions (each comprising10 min of pumping per breast per session) until initiation of direct breastfeeding. After the infants were able to suck, the usage of breast pump was restricted to remove excessive breast milk, to alleviate the fullness of breast milk, to donate breast milk, or to discard breast milk likely to be contaminated with medications. The number of sessions of breast pumping per day was expected to decrease gradually as lactation became established in healthy mother-infant dyads.

The 10-minute expression volume of breast milk per breast at 3 days and at 6 weeks postpartum were recorded, and the mother-infant dyads were followed up at 4 and 6 months after birth (Figure 1).

All women in this study would be at hospital more than 48 hrs after delivery and discharge at the $3^{\text {rd }}$ day. At the $3^{\text {rd }}$ day, the two breasts were sequentially or simultaneously pumped for 10 minutes. This was 
repeated 2-3 hours later, using the same type of pumping (Figure 1).

On a follow-up about day 6 weeks postpartum, the mothers were asked to breastfeed directly first, and one hour later each breast was pumped for 10 minutes. After a clinical review, the mothers were asked to breastfeed their infants directly again, and then have milk pumped with the type of breast pump crossed over one hour after second direct breastfeeding.

The participants also joined an online trial member group through WeChat (Tencent, China), a mobile phone App that facilitates instant communication between participants and investigators. One investigator was assigned to answer questions from the trial group and provide professional advice. The information on breastfeeding at 4 and 6 months were collected through online questionnaire.

\section{Outcomes and data collection}

The two primary outcomes were (1) Time for lactation initiation: also known as time for breastfeeding initiation, defined as the time of the infant was provided successfully direct breastfeeding or with expressed breast milk between delivery and hospital discharge and (2) Volume or weight of expressed breast milk in 20 min (10 min per breast) at three days or six weeks postpartum. To assess the effects of manual breast expression, milk transfer was measured at 3 days and at 6 weeks postpartum. The infants were weighed just before and after breastfeeding to determine milk transfer to the infant in the obstetrician ward. This method had been validated and was used in lactogenesis research ${ }^{23,49}$. Test weights of infants in cradle were obtained in duplicate using an electronic scale (accuracy $=0.1 \mathrm{~g}$ ). Each weight was recorded when the measurement was stable for 10 seconds. A density factor of $1.03 \mathrm{~g} / \mathrm{ml}$ was used in conversion between weight and volume of breast milk.

The secondary outcomes were the rates of exclusive breastfeeding at 4 and 6 months. (1) times of milk ejection during a expressing session (counted by mothers and nurse); [time frame: 6 weeks] (2) Breast milk production and mode of breastfeeding; the mode of breastfeeding divided into direct breastfeeding; direct breastfeeding+expressed breast milk; direct breastfeeding+expressed breast milk+formula milk powder; direct breastfeeding+formula milk powder; merely expressed breast milk; expressed breast milk+formula milk powder; totally formula milk powder; these data will be collected online; [time frame: six months]. Breastfeeding initiation was defined as the appearance of colostrum following breast expression or infant's sucking, and thus the time between delivery and colostrum appearance was defined to be the time required for breastfeeding initiation.

The reported adverse events during breast pumping include those from mothers and from device. General adverse events are those which patients or trial participants have complained about or clinicians have observed.

\section{Statistical analysis}


According to unpublished data from a pilot comparison between two groups of lactation-established women that each applied one of the two types of breast pumps, there was a difference in breast milk volume of around $15 \mathrm{ml}$ with a standard deviation of $40 \mathrm{ml}$. The sample size was then estimated to be 90 participants in each group with statistical power of 0.8 and significant level of 0.05 . Considering the limit of eligible participants, the sample size was adjusted to 50 participants in each group, and a cross over design of breast pump types was used at 6 weeks.

The data were analysed according to intention-to-treat (ITT). The continuous data were presented with mean $\pm S D$ and the categorical data were presented as count and percentage. To compare baseline characteristics, two-tailed one-way ANOVA was conducted for variables with a normal distribution or the Wilcoxon signed rank (Mann-Whitney $\mathrm{U}$ ) test for those not. Among three trial groups, nonparametric Kruskal-Wallis rank sum test and chi-squared test were applied for continuous and categorical data, respectively. Tukey honest significant differences were calculated in multiple comparison adjusted with $p$ value. Paired student's t test was applied to compare the milk volume between left and right breast or between two breast pumps. The log-rank test was applied to compare the time required for breastfeeding initiation (event=1). Linear mixed-effect modelling (Ime4 package) was used to analyze the association between the expressed milk volume and co-variates. $p<0.05$ was considered statistically significant. Statistical analyses were done with R(version 3.3.3)..$^{50}$

\section{Results}

\section{Study participants}

Following prenatal examination 226 participants returned written informed consent. After delivery $2 / 3$ of them $(n=154)$ were randomized into three groups of breast expression. Six participants withdrew between hospital discharge and review at 6 weeks. Meanwhile 2 participants stopped breastfeeding prematurely due to maternal health problems. Afterwards over $20 \%$ participants declined feedback at 4 months though they remained in contact. At 6 months less than half of participants in total remained in contact. More participants lost in follow-up during the last 2 months than in previous 4 months, especially in group of sequential breast expression (Figure 1). The three groups were well balanced at baseline (Table 1).

\section{Primary outcomes}

The method of breast milk expression on the time required for breastfeeding initiation was a primary outcome measure. The estimated median time that was required for breastfeeding initiation of hand expression, sequential breast pumping, and simultaneous breast pumping were 46.7 ( $95 \% \mathrm{Cl} 40.8-50.0)$, 46.5 (95\% $\mathrm{Cl} 36.1-50.5)$ and 45.6 (95\% Cl 34.2-53.0) hours, respectively. Each of 3 groups had around $10 \%$ participants that initiated breastfeeding after 3 days $(>72 \mathrm{hr}$ ). Compared with hand expression, sequential and simultaneous breast pumping did not promote earlier initiation of breastfeeding (log-rank test, $p=1.0$ ) (Figure 2A). For dyads with vaginal delivery, the median time to breastfeeding initiation with 
simultaneous breast pumping was 34.2 (95\% $\mathrm{Cl} 26.9-53.0)$ hours, with sequential pumping is 45.3 ( $95 \%$ $\mathrm{Cl}$ 20.3-49.0) hours, and with hand expression 45.7 (95\% Cl 31.4-51.8) hours. For dyads in which cesarean section had been used, the median times to initiation with breast pumps were somewhat longer - 49.3 (95\% Cl 38.9-66.8, p=0.096) hours for simultaneous pumping and 50.5 (95\% Cl 40.2-67.1, p=0.086) hours for sequential pumping. However, the differences were not statistically significant (Figure 2B). Comparing the time between two delivery modes, the time required for breastfeeding initiation in women with vaginal delivery was significantly earlier (median 45.1 (95\% Cl 31.4-48.0) hours) than in women who had cesarean section (median 48.2 (95\% Cl 43.5-53.0) hours; $p=0.0016$ ) (Figure $2 \mathrm{C}$ ).

The efficacy of breast expression in early and mature stages of lactation was measured through expressed breast milk volume. At 3 days after birth the infants in hand expression group were breastfed directly and weighed right before and after feeding. The expressed breast milk was also weighed. Averaged breast milk volumes were $23.8 \pm 26.2 \mathrm{ml}$ for hand expression, $16.7 \pm 11.6 \mathrm{ml}$ for sequential pumping and $13.1 \pm 10.9 \mathrm{ml}$ for simultaneous pumping. Averaged breast milk volume in simultaneous pumping was less than that in hand expression group through direct breastfeeding $(p=0.0073)$. However, it was not different from that for sequential pumping $(p=0.56)$. There was no difference between two delivery modes (vaginal delivery vs cesarean section) and no difference in subgroups of delivery mode in three groups (Figure 3A).

At 6 weeks the averaged breast milk volumes were $89.8 \pm 67.3 \mathrm{ml}$ of hand expression, $64.7 \pm 51.0 \mathrm{ml}$ of sequential pumping and $71.6 \pm 50.8 \mathrm{ml}$ of simultaneous pumping in a 10 -minute session. The average breast milk volume of sequential pumping was statistically less than that of hand expression $(p=0.035)$ but was not different from that of simultaneous pumping $(p=0.67)$. The averaged breast milk volume in participants with different delivery modes was not different $(p=0.834)$, with $72.8 \pm 55.1 \mathrm{ml}$ in vaginal delivery and $71.8 \pm 55.0 \mathrm{ml}$ in cesarean section group. Further subgroup analysis of breast milk volume of delivery modes in three groups did not show any statistical difference (Figure 3B). The average milk volume from left breast was about $2 \mathrm{ml}$ less than those from right breast $(p=0.012)$.

To minimize random effect from individual woman, the design of two breast pump types by two periods $(2 \times 2)$ cross over was applied at 6 weeks. In 1 st period (before breast pump cross over) the average breast milk volumes of sequential and simultaneous pumping groups were $40.4 \pm 25.3 \mathrm{ml}$ and $76.5 \pm 52.5 \mathrm{ml}$ $(p=0.0009)$, respectively. In 2 nd period (after breast pump cross over) the averaged breast milk volumes of sequential and simultaneous pumping groups were $88.6 \pm 58.3 \mathrm{ml}$ and $67.0 \pm 49.7 \mathrm{ml}(p=0.167)$, respectively. Total breast milk volume pumped in $1 \mathrm{st}$ period $(59.3 \pm 45.0 \mathrm{ml})$ was less than that in $2 \mathrm{nd}$ period $(79.0 \pm 55.1 \mathrm{ml})(p=0.0094)$. The breast milk volume increased from sequential to simultaneous pumping transition was statistically significant $(p<0.001)$, while the converse simultaneous to sequential transition was not (Figure 4). Next, a linear mixed effects model was fitted for this cross over design by using breast milk volume at 6 weeks as the dependent variable. The predictive value of breast milk volume at 3 days was negligible by using a backward elimination for linear mixed models (ImerTest package). After restricted likelihood ratio tests on simulated values (RLRsim package), the estimated model coefficients indicated that the breastfeeding initiation time would affect the breast milk volume 
inversely and sequential pumping was inferior to simultaneous pumping in efficacy of breast milk expression (Table 2).

\section{Secondary outcomes}

At 4 months the averaged body weight and length of infants were $7429.9 \pm 1253.4 \mathrm{~g}$ and $63.4 \pm 10.5 \mathrm{~cm}$, respectively. Median count of self-reported direct breastfeeding was 5.5 (interquartile range, IQR 2-8) sessions each day, median of count of using breast pump was 2 (IQR 2-4) sessions per day, and the averaged breast milk volume expressed each session was $172.4 \pm 95.7 \mathrm{ml}$ in responsive women. In the following two months the body weight increased by about 800 grams $(p<0.0001)$ and body length increased by $3 \mathrm{~cm}(p=0.0298)$ on average. The baby's body weights differed between the three groups at six months $(p=0.0163)$ while their body lengths differed between the three groups at four months $(p=0.0326)$. Except that there was no difference in three groups in recorded feedbacks. In two breast pumping groups, $80 \%$ of mothers used breast pumps frequently. The paired student t test on expressed milk volume of mothers showed no difference $(p=0.787)$ between their response at 4 and at 6 months (pairs=41). The expressed breast milk volume in mothers who responded only once (at 4 months, $n=35$ ) was less than that responded twice (at 4 and 6 months, $n=41$ ), with $136.8 \mathrm{ml}$ vs $202.6 \mathrm{ml}, p=0.0024$. About $90 \%$ mothers supplemented food for their infants at 6 months. Absence of milk, milk insufficiency, or return-to-work might explain why the rate of introduction of formula milk was $40 \%$ - at 4 and $50 \%$ at 6 months. In terms of breastfeeding mode, the proportion of exclusive breastfeeding dropped from $21 \%$ at 4 months to $10 \%$ at 6 months, although this was not statistically significant (Table 3).

\section{Adverse events}

No serious or severe adverse events episodes were reported in follow-up to six months. The proportion of all types of local breast adverse events was $11.5 \%$ and of all types of systemic adverse events of mild to moderate severity was $2.9 \%$. Electric breast pump-associated adverse events localized to the breast included erythema, rash, lump, swelling, nipple sore, chapping and peeling and those that appeared to be systemic responses requiring medical intervention included mastitis and erysipelas.

\section{Discussion}

In this open-label, randomized control trial, we studied different methods of breast expression immediately after birth in the absence of infant sucking. The time required for breastfeeding initiation in the three groups - hand expression, sequential electric pumping, and simultaneous electrical pumping was not statistically different. The 10 -minute breast milk volume extracted through breast pumps was less than that of direct breastfeeding. Compared with participants with vaginal delivery, the time required for breastfeeding initiation was delayed in those with cesarean section, however, there was no significant difference in breast milk volume at 3 days and 6 weeks. Further analysis indicated that the time required for breastfeeding initiation may inversely predict expressed breast milk volume at 6 weeks. The efficacy of sequential pumping was inferior to that of simultaneous pumping in the 10-minute period. The rates of exclusive breastfeeding at 4 and 6 months were not different in three groups. 


\section{Comparison with other studies}

Breastfeeding initiation within $1 \mathrm{hr}$ after birth may facilitate the appearance of copious breast milk volume $22-24,51$ and reduce risk of infant death in first 28 days. ${ }^{52}$ Early skin-to-skin contact, mode of delivery, and maternal illness were associated with early breastfeeding initiation. ${ }^{53,54}$ For participants of immediate mother-and-infant separation after birth, they also had other conditions that require medical care. That made introduction of breast expression within $1 \mathrm{hr}$ not always practical. We introduced breast expression as soon as possible after birth. This was intended to promote an early breastfeeding initiation for the dyads who were separated more than $6 \mathrm{hrs},{ }^{22}$ as breast expression at 24-72 hrs after cesarean section would not improve milk transfer. ${ }^{23}$ Alternatively, antenatal breast expressing (twice daily at convenient time) after 37 weeks of gestational age was recommended ${ }^{55-58}$ and trial was taken recently in women with diabetes in pregnancy. ${ }^{59}$ In the present trial, the time for breastfeeding initiation ranged from $17 \mathrm{hrs}$ before delivery to $95 \mathrm{hrs}$ after delivery, with median time greater than $45 \mathrm{hrs}$ in all six subgroups of expression methods and delivery mode. This suggests some common underlying causes had influence on both the mother-infant separation after birth and timing of lactation initiation. In line with this delayed breastfeeding initiation, the median time between delivery and sudden copious breast milk (self-reported data to indicate start of lactogenesis stage II) ranged from 4.0 to7.5 days. Usually, the lactogenesis stage II would start at $30-48 \mathrm{hrs}$, and delayed lactogenesis stage II (>72 hrs) would happen in $22 \%-31 \%$ of mothers. ${ }^{60,61}$

\section{Clinical relevance}

Breast milk transfer in the hand expression group under specified conditions, such as maternal-baby separation by delivery complications, could be viewed as comparable to that in healthy infants of direct breastfeeding in general population. At 3 days, the averaged breast milk volume through pumping was less than those from hand expressing though only those in simultaneous pumping group was significant. At 6 weeks the breast milk volume pumped simultaneously in the 10-minute period was much closer to that obtained through direct breastfeeding than to that pumped sequentially. Nevertheless, the average milk volume pumped was $18 \mathrm{ml}$ less than that of direct breastfeeding in the same period. Therefore, direct breastfeeding is undoubtedly the optimal option of breast milk extraction. Compared with sequential pumping, simultaneous breast pumping had an extra stimulation step (with 1.0-2.5 Hz and 80$150 \mathrm{mmHg}$ vacuum) before expression. The inconsistency of breast pumps in different study populations and timing indicated that the role of feedback control of breast milk extraction, if any, was limited. 22,25,45,62,63 The electric breast pumps should be viewed as a tool solely for breast milk expression when other methods are not available and should not interfere with breastfeeding practice.

\section{Comparison among the three method of breast expression}

The reasons for inadequate rate and duration of exclusive breastfeeding often result from factors including planned cesarean section delivery, ${ }^{64}$ gestational age at delivery, breast refusal, sore nipple, lack of access to breast pumps, use of free formula milk, ${ }^{37,41,63,65}$ insufficient support for breastfeeding, self- 
perceived inadequacy of milk supply, having to leave infants to go to work or shcool ${ }^{19,39,40,66,67}$ and medical conditions ${ }^{45}$. Among participants who responded, $80.0 \%$ used breast pumps at 4 months and $71.9 \%$ at 6 months. The proportions were not different among three groups at 4 and 6 months $(p=0.079$, $p=0.436)$, or by delivery modes ( $p=0.737)$. At 4 months, the maternity leave of $50.4 \%$ nursing mothers had ended, and this rose to $80.0 \%$ at 6 months $(p=0.0002)$. However, the proportion of breast pump usage in maternity leave was not different from those out of maternity leave at 4 months $(p=1.0)$, or at 6 months $(p=0.691)$. We do not know the reasons for application of breast pumps before 6 months, however, the high rate of breast pump usage could match the proportion of exclusive breastfeeding quite well inversely.

\section{Limitations of the study}

The results of this trial should be interpreted with caution because its specific study population selected mother-infant separation immediately after birth. Women in trial groups with sufficient breast milk supply would be more likely to respond at 4 and 6 months. The high rate of breast pumping among nursing women, instead of laborious hand expressing, would lead to biased estimation of breast milk volume as the effect of breast pump on breast milk production yet to be justified.

\section{Conclusions}

Introducing breast expression (hand expressing, sequential and simultaneous pumping) in the period of mother-infant-separation since 2 hrs postpartum did not display different effects on time for breastfeeding initiation. Simultaneous breast pumping (in this case) was more effective than sequential pumping in breastfeeding established women both in volume and time while the direct breastfeeding was optimal in breast milk extraction at 3 days and 6 weeks. The time for breastfeeding initiation could predict the volume of pumped breast milk at 6 weeks inversely. Three methods of breast expression did not affect the rates of exclusive breastfeeding at 4 and 6 months.

\section{List Of Abbreviations}

WHO: World Health Organization; CONSORT statement; ITT: Intention-to-treat; one-way ANOVA: One-factor Analysis of Variance; IQR: Interquartile range; BMI: Body mass index; EBF: Exclusive direct breastfeeding; $\mathrm{BF}+$ : Breastfeeding directly or with sucked milk plus other food including milk powder.

\section{Declarations}

\section{Ethics approval and consent to participate}

All participants in this study provided written informed consent before recruitment, and the Institutional Review Board of Xinhua hospital approved this project (XHEC-C2017-108-2). 
Not applicable.

\section{Availability of data and materials}

The datasets used and/or analyzed during the current study are available from the corresponding author on reasonable request.

\section{Competing interests}

The authors declare that they have no competing interests.

\section{Funding}

This study was supported in part by funds granted to Lin Zhang from: the Shanghai Hospital development center (SHDC12016204), the Shanghai Municipal Health Bureau (201640032), and the Xinhua Hospital Fund (18JX002). Philips Invest (China) Co. Ltd. provided the breast pumps and pilot study data on breast milk volume difference for sample size estimation.

\section{Authors' Contributors}

LZ and JYN designed the study. LZ coordinated the study. YZ and JYN enrolled the participants and arranged informed consent from the participants and drafted of the manuscript. LJW and CCZ provided care for participants and checked the questionnaires. YC and CCZ conducted the test weighing and checked the breast milk volume and conducted the breast pump types cross over. LZ and YZ did the data analyses including statistical analyses. All authors critically reviewed the report. LZ had full access to all of the data in this study and take responsibility for the integrity of the data and accuracy of the data analyses.

\section{Acknowledgements}

We are grateful to research staff for help with data collection in participating institutions.

\section{References}

1. Victora CG, Bahl R, Barros AJ, França GV, Horton S, Krasevec J, et al. Breastfeeding in the 21st century: epidemiology, mechanisms, and lifelong effect. Lancet 2016; 387(10017): 475-90.

2. Harding JE, Cormack BE, Alexander T, Alsweiler JM, Bloomfield FH. Advances in nutrition of the newborn infant. Lancet 2017; 389(10079): 1660-8.

3. WHO. Global strategy for infant and young child feeding. 2003.

https://www.who.int/nutrition/publications/gs_infant_feeding_text_eng.pdf (accessed 2019-06-25 2019).

4. Edmond KM, Zandoh C, Quigley MA, Amenga-Etego S, Owusu-Agyei S, Kirkwood BR. Delayed breastfeeding initiation increases risk of neonatal mortality. Pediatrics 2006; 117(3): e380-e6. 
5. Black RE, Victora CG, Walker SP, Bhutta ZA, Christian P, de Onis M,et al. Maternal and child undernutrition and overweight in low-income and middle-income countries. Lancet 2013; 382(9890): 427-51.

6. Debes AK, Kohli A, Walker N, Edmond K, Mullany LC. Time to initiation of breastfeeding and neonatal mortality and morbidity: a systematic review. BMC Public Health 2013; 13 Suppl 3: S19-S.

7. Pattison KL, Kraschnewski JL, Lehman E,Savage JS, Downs DS, Leonard KS, et al. Breastfeeding initiation and duration and child health outcomes in the first baby study. Prev Med 2019; 118: 1-6.

8. Jones ME, Swerdlow AJ, Gill LE, Goldacre MJ. Pre-natal and early life risk factors for childhood onset diabetes mellitus: a record linkage study. Int J Epidemiol 1998; 27(3): 444-9.

9. Grummer-Strawn LM, Mei Z, Control CfD, System PPNS. Does breastfeeding protect against pediatric overweight? Analysis of longitudinal data from the Centers for Disease Control and Prevention Pediatric Nutrition Surveillance System. Pediatrics 2004; 113(2): e81-e6.

10. Rich-Edwards JW, Stampfer MJ, Manson JE, Rosner B, Hu FB, Michels KB,et al. Breastfeeding during infancy and the risk of cardiovascular disease in adulthood. Epidemiology 2004; 15(5): 550-6.

11. Heikkilä K, Kelly Y, Renfrew MJ, Sacker A, Quigley MA. Breastfeeding and educational achievement at age 5. Matern Child Nutr 2014; 10(1): 92-101.

12. Whittemore AS, Harris R, Itnyre J. Characteristics relating to ovarian cancer risk: collaborative analysis of 12 US case-control studies. II. Invasive epithelial ovarian cancers in white women. Collaborative Ovarian Cancer Group. Am J Epidemio/ 1992; 136(10): 1184-203.

13. Labbok MH. Effects of breastfeeding on the mother. Pediatr Clin North Am 2001; 48(1): 143-58.

14. Cancer CGoHFiB. Breast cancer and breastfeeding: collaborative reanalysis of individual data from 47 epidemiological studies in 30 countries, including 50302 women with breast cancer and 96973 women without the disease. Lancet 2002; 360(9328): 187-95.

15. Sobhy SI, Mohame NA. The effect of early initiation of breast feeding on the amount of vaginal blood loss during the fourth stage of labor. J Egypt Public Health Assoc 2004; 79(1-2): 1-12.

16. Modugno F, Goughnour SL, Wallack D, Edwards RP, Odunsi K, Kelley JL, et al. Breastfeeding factors and risk of epithelial ovarian cancer. Gynecol Oncol 2019; 153(1): 116-22.

17. Roberts TJ, Carnahan E, Gakidou E. Can breastfeeding promote child health equity? A comprehensive analysis of breastfeeding patterns across the developing world and what we can learn from them. BMC Med 2013; 11: 254-.

18. Besore CT. Barriers to breastfeeding for Hispanic mothers. Breastfeed Med 2014; 9(7): 352-4.

19. Loewenberg Weisband Y, Keim SA, Keder LM, Geraghty SR, Gallo MF. Early Breast Milk Pumping Intentions Among Postpartum Women. Breastfeed Med 2017; 12: 28-32.

20. Parker LA, Sullivan S, Krueger C, Kelechi T, Mueller M. Effect of early breast milk expression on milk volume and timing of lactogenesis stage II among mothers of very low birth weight infants: a pilot study. J Perinatol 2012; 32(3): 205-9. 
21. Hill PD, Aldag JC, Chatterton RT, Zinaman M. Comparison of milk output between mothers of preterm and term infants: the first 6 weeks after birth. J Hum Lact 2005; 21(1): 22-30.

22. Parker LA, Sullivan S, Krueger $C$, Mueller M. Association of timing of initiation of breastmilk expression on milk volume and timing of lactogenesis stage II among mothers of very low-birthweight infants. Breastfeed Med 2015; 10(2): 84-91.

23. Chapman DJ, Young S, Ferris AM, Pérez-Escamilla R. Impact of breast pumping on lactogenesis stage II after cesarean delivery: a randomized clinical trial. Pediatrics 2001; 107(6): E94-E.

24. Meier PP, Engstrom JL, Janes JE, Jegier BJ, Loera F. Breast pump suction patterns that mimic the human infant during breastfeeding: greater milk output in less time spent pumping for breast pumpdependent mothers with premature infants. J Perinato/2012; 32(2): 103-10.

25. Rowe-Murray HJ, Fisher JRW. Baby friendly hospital practices: cesarean section is a persistent barrier to early initiation of breastfeeding. Birth 2002; 29(2): 124-31.

26. Zanardo V, Svegliado G, Cavallin F, Giustardi A, Cosmi E, Litta P, et al. Elective cesarean delivery: does it have a negative effect on breastfeeding? Birth 2010; 37(4): 275-9.

27. Zhang F, Yang Y, Bai T, Sun L, Sun M, Shi X, et al. Effect of pumping pressure on onset of lactation after caesarean section: A randomized controlled study. Matern Child Nutr 2018; 14(1).

28. Prior E, Santhakumaran S, Gale C, Philipps LH, Modi N, Hyde MJ. Breastfeeding after cesarean delivery: a systematic review and meta-analysis of world literature. Am J Clin Nutr 2012; 95(5): 111335.

29. Wallenborn JT, Masho SW. The Interrelationship Between Repeat Cesarean Section, Smoking Status, and Breastfeeding Duration. Breastfeed Med 2016; 11: 440-7.

30. Zhao J, Zhao Y, Du M, Binns CW, Lee AH. Does Caesarean Section Affect Breastfeeding Practices in China? A Systematic Review and Meta-Analysis. Matern Child Health J 2017; 21(11): 2008-24.

31. Hakala M, Kaakinen P, Kääriäinen M, Bloigu R, Hannula L, Elo S. The realization of BFHI Step 4 in Finland - Initial breastfeeding and skin-to-skin contact according to mothers and midwives. Midwifery 2017; 50: 27-35.

32. Chen C, Yan Y, Gao X, Xiang S, He Q, Zeng G, et al. Influences of Cesarean Delivery on Breastfeeding Practices and Duration: A Prospective Cohort Study. J Hum Lact 2018; 34(3): 526-34.

33. Zhang F, Cheng J, Yan S, et al. Early Feeding Behaviors and Breastfeeding Outcomes After Cesarean Section. Breastfeed Med 2019; 14(5): 325-33.

34. Li HT, Luo S, Trasande L, Wu H, Bai T. Geographic Variations and Temporal Trends in Cesarean Delivery Rates in China, 2008-2014. JAMA 2017; 317(1): 69-76.

35. Rollins NC, Bhandari N, Hajeebhoy N, Horton S, Lutter CK, Martines JC, et al. Why invest, and what it will take to improve breastfeeding practices? Lancet 2016; 387(10017): 491-504.

36. Dougherty D, Luther M. Birth to breast--a feeding care map for the NICU: helping the extremely low birth weight infant navigate the course. Neonatal Netw 2008; 27(6): 371-7. 
37. Flaherman VJ, Lee HC. "Breastfeeding" by feeding expressed mother's milk. Pediatr Clin North Am 2013; 60(1): 227-46.

38. Dinour LM, Pope GA, Bai YK. Breast milk pumping beliefs, supports, and barriers on a university campus. J Hum Lact 2015; 31(1): 156-65.

39. Labiner-Wolfe J, Fein SB, Shealy KR, Wang C. Prevalence of breast milk expression and associated factors. Pediatrics 2008; 122 Suppl 2: S63-S8.

40. Johns HM, Amir LH, McLachlan HL, Forster DA. Breast pump use amongst mothers of healthy term infants in Melbourne, Australia: A prospective cohort study. Midwifery 2016; 33: 82-9.

41. Jiang B, Hua J, Wang Y, Fu Y, Zhuang Z, Zhu L. Evaluation of the impact of breast milk expression in early postpartum period on breastfeeding duration: a prospective cohort study. BMC Pregnancy Childbirth 2015; 15: 268-.

42. Felice JP, Rasmussen KM. Breasts, Pumps and Bottles, and Unanswered Questions. Breastfeed Med 2015; 10(9): 412-5.

43. Felice JP, Geraghty SR, Quaglieri CW, Yamada R, Wong AJ, Rasmussen KM. "Breastfeeding" but not at the breast: Mothers' descriptions of providing pumped human milk to their infants via other containers and caregivers. Matern Child Nutr 2017; 13(3).

44. Forster DA, Johns HM, McLachlan HL, Moorhead AM, McEgan KM, Amir LH. Feeding infants directly at the breast during the postpartum hospital stay is associated with increased breastfeeding at 6 months postpartum: a prospective cohort study. BMJ Open 2015; 5(5): e007512-e.

45. Bream E, Li H, Furman L. The Effect of Breast Pump Use on Exclusive Breastfeeding at 2 Months Postpartum in an Inner-City Population. Breastfeed Med 2017; 12: 149-55.

46. Becker GE, Smith HA, Cooney F. Methods of milk expression for lactating women. Cochrane Database Syst Rev 2016; 9: CD006170-CD.

47. Juszczak E, Altman DG, Hopewell S, Schulz K. Reporting of Multi-Arm Parallel-Group Randomized Trials: Extension of the CONSORT 2010 Statement. JAMA 2019; 321(16): 1610-20.

48. Breastfeeding: a guide for medical profession. 8th ed. Philadelphia PA, USA: Elsevier; 2015.

49. Neville MC, Keller R, Seacat J, Lutes V, Neifert M, Casey C, et al. Studies in human lactation: milk volumes in lactating women during the onset of lactation and full lactation. Am J Clin Nutr 1988; 48(6): 1375-86.

50. Team RC. R: A Language and Environment for Statistical Computing. 3.3.3 ed. Vienna, Austria: R Foundation for Statistical Computing; 2017.

51. Karim F, Billah SM, Chowdhury MAK, Zaka N, Manu A, Arifeen SE, et al. Initiation of breastfeeding within one hour of birth and its determinants among normal vaginal deliveries at primary and secondary health facilities in Bangladesh: A case-observation study. PLoS One 2018; 13(8): e0202508-e.

52. Group NEOVITAS. Timing of initiation, patterns of breastfeeding, and infant survival: prospective analysis of pooled data from three randomised trials. Lancet Glob Health 2016; 4(4): e266-e75. 
53. Lau Y, Tha PH, Ho-Lim SST, Wong LY, Lim PI, Citra Nurfarah BZM, et al. An analysis of the effects of intrapartum factors, neonatal characteristics, and skin-to-skin contact on early breastfeeding initiation. Matern Child Nutr 2018; 14(1).

54. Sallam SA, Babrs GM, Sadek RR, Mostafa AM. Knowledge, attitude, and practices regarding early start of breastfeeding among pregnant, lactating women and healthcare workers in El-Minia University Hospital. Breastfeed Med 2013; 8(3): 312-6.

55. Singh G, Chouhan R, Sidhu K. Effect of Antenatal Expression of Breast Milk at Term in Reducing Breast Feeding Failures. Med J Armed Forces India 2009; 65(2): 131-3.

56. Cox S. An ethical dilemma: should recommending antenatal expressing and storing of colostrum continue? Breastfeed Rev 2010; 18(3): 5-7.

57. Chapman T, Pincombe J, Harris M, Fereday J. Antenatal breast expression: exploration and extent of teaching practices amongst International Board Certified Lactation Consultant midwives across Australia. Women Birth 2013; 26(1): 41-8.

58. East CE, Dolan WJ, Forster DA. Antenatal breast milk expression by women with diabetes for improving infant outcomes. Cochrane Database Syst Rev 2014; (7): CD010408-CD.

59. Forster DA, Moorhead AM, Jacobs SE, Davis PG, Walker SP, McEgan KM, et al. Advising women with diabetes in pregnancy to express breastmilk in late pregnancy (Diabetes and Antenatal Milk Expressing [DAME]): a multicentre, unblinded, randomised controlled trial. Lancet 2017; 389(10085): 2204-13.

60. Breastfeeding So. Breastfeeding and the use of human milk. Pediatrics 2012; 129(3): e827-e41.

61. Zhu P, Hao J, Jiang X, Huang K, Tao F. New insight into onset of lactation: mediating the negative effect of multiple perinatal biopsychosocial stress on breastfeeding duration. Breastfeed Med 2013; 8: 151-8.

62. Wagner S, Kersuzan C, Gojard S, Tichit C, Nicklaus S, Thierry X, et al. Breastfeeding initiation and duration in France: The importance of intergenerational and previous maternal breastfeeding experiences - results from the nationwide ELFE study. Midwifery 2019; 69: 67-75.

63. Win NN, Binns CW, Zhao Y, Scott JA, Oddy WH. Breastfeeding duration in mothers who express breast milk: a cohort study. Int Breastfeed J 2006; 1: 28.

64. Liu X, Zhang J, Liu Y, Li Y, Li Z. The association between cesarean delivery on maternal request and method of newborn feeding in China. PLoS One 2012; 7(5): e37336-e.

65. Chantry CJ. Supporting the 75\%: overcoming barriers after breastfeeding initiation. Breastfeed Med 2011; 6: 337-9.

66. Lin SY, Lee JT, Yang CC, Gau ML. Factors related to milk supply perception in women who underwent cesarean section. J Nurs Res 2011; 19(2): 94-101.

67. Clemons SN, Amir LH. Breastfeeding women's experience of expressing: a descriptive study. J Hum Lact 2010; 26(3): 258-65. 


\section{Tables}

Please see the supplementary files section to view the tables.

\section{Figures}

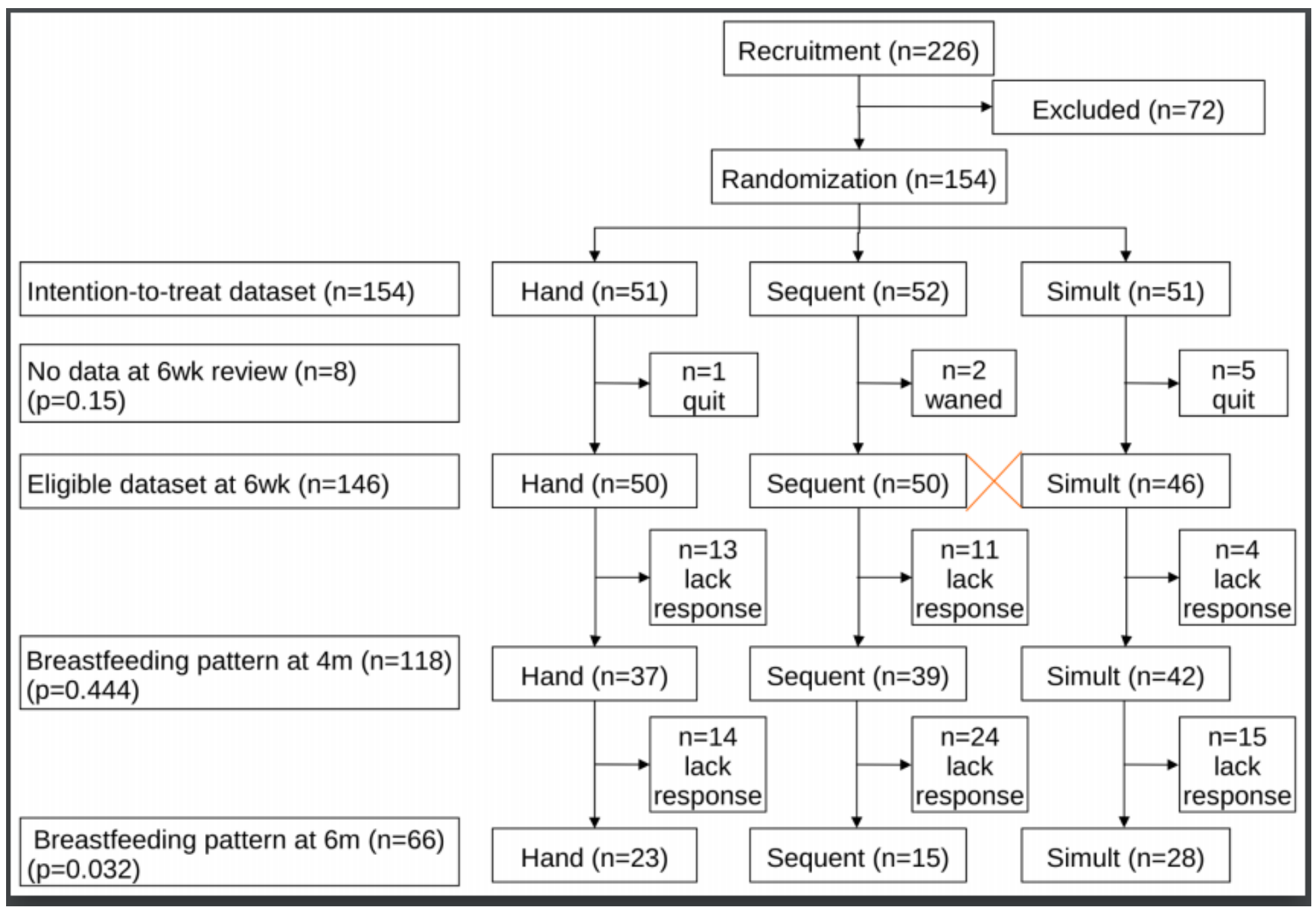

Figure 1

Participants flowchart Hand stands for hand expression if required and the infants were breastfed directly; sequent stands for sequential breast pumping; simult stands for simultaneous breast pumping; an inset graph for detailed cross over of breast pumps was also provided. 


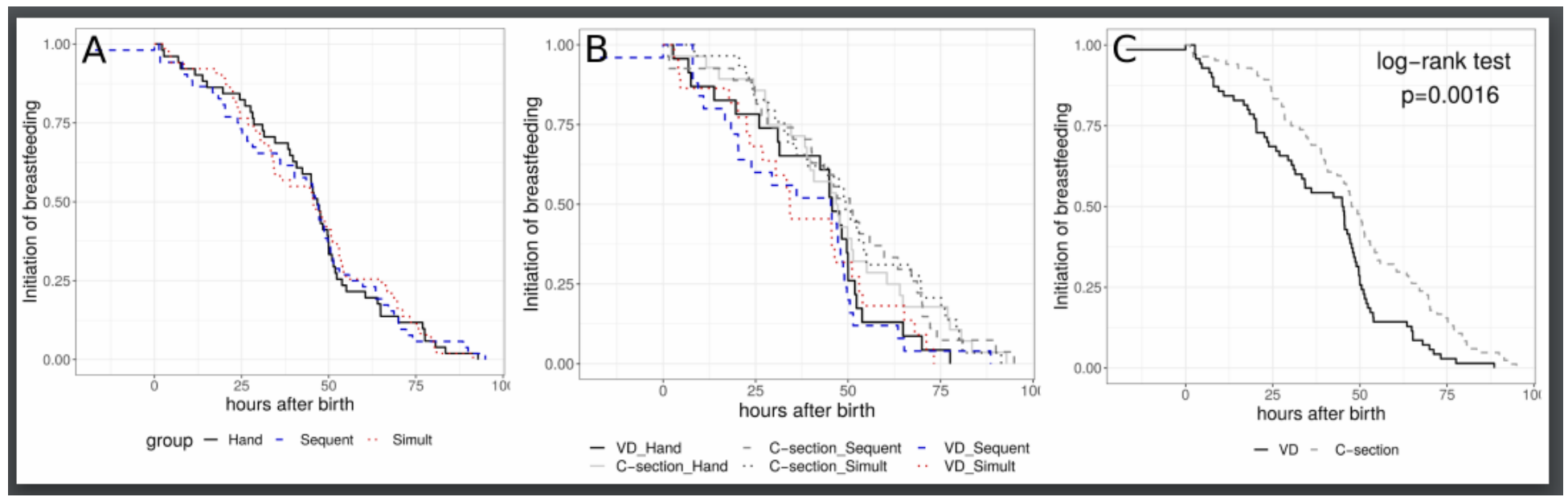

Figure 2

Time for breastfeeding initiation in survival analysis Breastfeeding initiation, also known as colostrum appearance in this study, was viewed as an event; successful breastfeeding initiation defined as events=1; VD, vaginal delivery; C-sect, cesarean section; (A) breastfeeding initiation in hand expression, sequential and simultaneous pumping; (B) breastfeeding initiation in subgroups of delivery mode (VD vs C-sect) of three groups; (C) comparison between two delivery mode across all three groups;

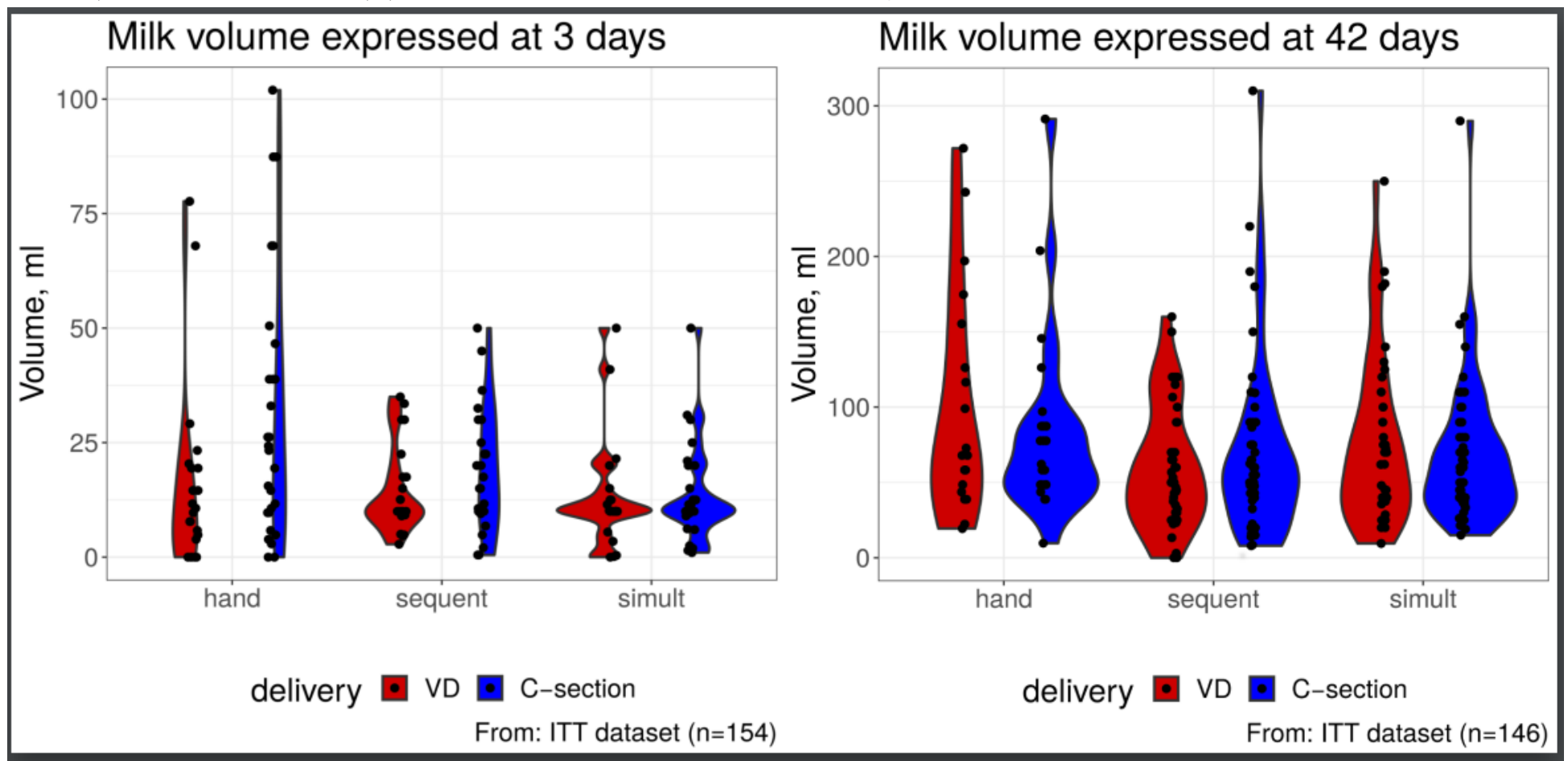

Figure 3

Breast milk volume expressed at 3 days and 6 weeks Breast milk volume in hand expression group was estimated through test weighing of transferred milk through infants' sucking, strictly speaking it was not expressed; the mere difference in three groups were the methods of breast expression without infant sucking, the infant should be direct breastfed or indicated otherwise; 


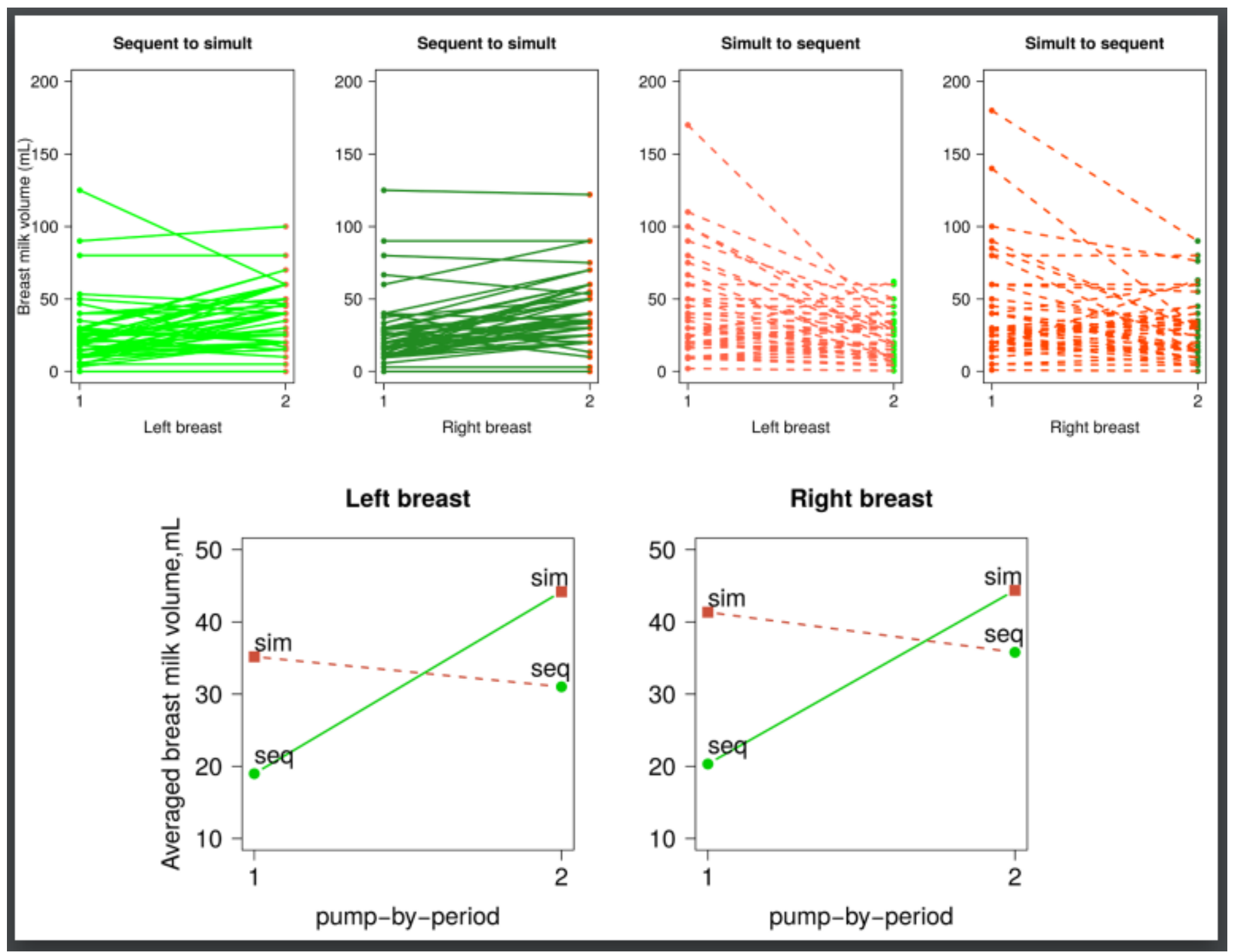

Figure 4

Breast pumps cross over at 6 weeks The upper panel displayed the breast milk volume in two periods of pumping in individual in two different pattern of shift, namely from sequential to simultaneous pumping or vice versa; the green dots represented the sequential pumping, the orange dots for simultaneous pumping; the lower panel provided the averaged levels of each groups in each period; sim, simultaneous pumping; seq, sequential pumping;

\section{Supplementary Files}

This is a list of supplementary files associated with this preprint. Click to download.

- Tables.docx

- clinitaltrialprotocolofABPfinalv32017En.pdf

- CONSORT2010ChecklistMSWord.doc 
- SFigure01breastexp3workflowchartinset201910.pdf 